\title{
Real space in situ bond energies: toward a consistent energetic definition of bond strength
}

\author{
D. Menéndez-Crespo, A. Costales, E. Francisco, and A. Martín Pendás ${ }^{a *}$ \\ June 20, 2018
}

\begin{abstract}
A rigorous definition of intrinsic bond strength based on the partitioning of a molecule into real space fragments is presented. Using the domains provided by the quantum theory of atoms in molecules (QTAIM) together with the interacting quantum atoms (IQA) energetic decomposition, we show how an in situ bond strength, matching all the requirements of an intrinsic bond energy, can be defined between each pair of fragments. Total atomization or fragmentation energies are shown to be equal to the sum of these in situ bond energies (ISBEs) if the energies of the fragments are measured with respect to their in-the-molecule state. These energies usually lie above the ground state of the isolated fragments by quantities identified with the standard fragment relaxation or deformation energies, which are also provided by the protocol. Deformation energies bridge dissociation energies with ISBEs, and can be dissected using well-known tools of real space theories of chemical bonding. Similarly, ISBEs can be partitioned into ionic and covalent contributions, and this feature adds to the chemical appeal of the procedure. All the energetic quantities examined are observable and amenable, in principle, to experimental determination. Several systems, exemplifying the role of each energetic term herein presented are used to show the power of the approach.
\end{abstract}

Keywords: bond energy, bond theory, QTAIM, IQA, energy decompositions.

\footnotetext{
${ }^{[a] D r . ~ D a n i e l ~ M e n e ́ n d e z-C r e s p o, ~ P r o f . ~ A u r o r a ~ C o s t a l e s, ~ P r o f . ~ E v e l i o ~ F r a n c i s c o, ~ P r o f . ~ A n g e l . ~ M a r t i ́ n ~ P e n d a ́ s . ~}$ Departamento de Química Física y Analítica.

Facultad de Química. Universidad de Oviedo.

33006-Oviedo. Spain.

E-mail: ampendas@uniovi.es
} 


\section{Introduction}

Despite being central to Chemistry, the concept of bond strength is still a matter of debate and legitimate scientific discussion. [1-3] At the core of the problem lies the non-measurability of bond energies in polyatomic molecules, [4] although discussions already flourish even in diatomics. [5-14] So important is the concept to Chemistry, and so deeply rooted it is in the chemist's mind that, even today, the role of empirical bond-length bond-strength correlations, like Badger's rule, $[15,16]$ are the aim of renewed discussions. $[17,18]$

Textbooks soon teach that the bond dissociation energy (BDE), the energy required to split a ground state molecule A-B into its fragments in their respective ground states measures bond strength. However, BDEs, which can be determined either experimentally or computationally, [4] may only be considered descriptors of bond energies (BEs) in diatomics.

In polyatomic molecules, where several independent bonds exist, BEs are not accessible. Moreover, reorganization processes accompanying dissociation, both geometrical and electronic, inevitably mingle with the true energy cost of bond breaking. A plethora of phenomena, ranging from the role of avoided crossings to charge transfer, the exacerbation of spin-orbit effects, the opening or closing of hyperconjugation or delocalization channels, the onset or destruction of aromatic character, etc, are seen by many researchers as difficulties to accept BDEs as reliable measures of bond strength.

For some of these researchers, [4, 19] only if all these effects are disentangled, i.e. only if we are able to obtain the energy cost of going from the ground state molecule to the fragments-in-themolecule, will we be able to define an appropriate intrinsic bond energy (IBE). Since these IBEs necessarily rest on more or less arbitrary prescriptions to define the fragments-in-the-molecule, there is not much hope in using energy derived bond strengths, others say. [18]

All these difficulties underlie the search and widespread use of non-energetic descriptors of bond strength. Three of them, bond lengths, force constants, and bond orders have found their way in the literature, and tens of different semiempirical bond-length bond-strength correlations have been proposed. [20] As Kaupp, Danovich and Shaik have recently noted, [18] the use of force constants ultimately rests on energy models of dissociation curves such as that of Morse, [21] for which a straightforward link between $D_{e}$ and the force constant $k_{e}$ exists. 
Work along these lines also faces severe obstacles. Normal mode coupling in polyatomics hampers the association of a particular vibration with the stretching of any given bond. This has led to the development of a number of theories of local vibrations, wherein the work of Konkoli and Cremer [22] on adiabatic modes stands out on its own. Almost two decades of work have shown very robust correlations, for instance, between adiabatic force constants, $k_{e}^{a}$ and relative bond orders (bond strength orders). It has also been suggested [4] that standard valence force field force constants, $k_{\mathrm{e}}^{\mathrm{c}}$, should be related to IBEs while adiabatic ones would correlate to BDEs.

Two recent works $[17,18]$ summarize the situation very clearly. For a school of thought, [17] there are so many dangers in using BDEs as bond strength parameters in the absence of reliable IBE indicators, that we are led to (local, adiabatic) force constants instead. For others, [18] "the chemical bond is inherently an energetic quantity" and restricting to quantities that sense only small displacements around minima to measure bond strength simply does not pay off. These authors also argue against IBEs which, besides their ambiguous definition, are not directly related to thermochemistry, advocating for turning back to BDEs.

Given this situation, we disclose in this contribution how the use of real space fragments can lead to a consistent energetic approach to bond strength. Once a molecule is properly divided into its constituent atoms or fragments, and this can be done at any nuclear configuration, e.g. at equilibrium but also at any point during dissociation, its energy can be written as a sum of atomic (or fragment) self-energies plus pair-wise additive interaction energies. As we will show, the former are nothing but the long-sought energies of the fragments-in-the-molecule, while the latter are IBEs. This in-the-molecule analysis provides intrinsic bonding information, and lacks any reference to the unbound reference fragments. To some, the consideration of reference fragments is essential to understand the origin of binding, [23] which is thought to be intimately linked to the changes that a molecule suffers upon dissociation.

Notwithstanding that different fragmentation channels may be of interest in different chemical environments, i.e. heterolytic versus homolytic rupture, and that intrinsic information is thus itself valuable, once a proper reference for the fragments has been chosen the BDE can also be written in our approach as a sum of so-called atomic (or fragment) deformation energies, the energy penalties taking us from the unbound fragments to the in-the-molecule ones, and the very same IBEs briefly sketched above. 
Deformation energies build the bridge linking IBEs to BDEs. In this sense, our protocol embraces both the intrinsic and the standard energetic views regarding bond strength, allowing an easy navigation between them. Deformation energies and IBEs can be dissected at different levels. As we will show, this kind of analysis gives valuable information about the origin of the deformation of the fragments. Since it can be done at any point on a potential energy surface, this allows for examining bonding for metastable systems. Similarly, IBEs can be dissected into ionic and covalent terms, providing a chemically intuitive framework.

The use of real space techniques guarantees the invariance of all our descriptors under orbital transformations, and thus assures their independence from any particular flavor of theory used to get them. Fragments are extracted from the quantum theory of atoms in molecules [24] (QTAIM), which rests on the electron density scalar, and energies from the interacting quantum atoms approach [25, 26] (IQA), which also needs the pair density. Although no efficient experimental technique has been devised to obtain the pair density as of today, the protocol here proposed is based on observables, and it is thus amenable to experimental determination. Actually, the quick progress of quantum crystallography [27] allows, already today, to recover our basic descriptors from experiment.

We will first recall briefly how bond energies are normally defined from bond dissociation energies. Then we introduce the QTAIM and IQA tools that form the nucleus of the in situ bond energies. A section on how to dissect and extract chemical information about the in-the-molecule states follows before examining a few systems that exemplify the role of the choice of reference states, the meaning of deformation energies in cases where fragment promotion has or has not been invoked in the past to understand binding, and the implications of the existence of ISBEs for each pair of fragments, chemically bonded or not.

\section{From bond dissociation energies to bond energies}

Bond dissociation energies for a general $A-B \rightarrow A+B$ process are determined either from: (i) calculated energy differences, $B D E=D_{e}=E(A)+E(B)-E(A-B)$, or (ii) from experimental standard dissociation enthalpies subjected to thermal corrections, in turn obtained from spectroscopic information of the parent A-B molecule and the dissociation fragments $A, B$. [28] We will disregard from now on the origin of BDEs. Given that our protocol will be exemplified from calculations, all follow- 
ing arguments will be done in terms of pure Born-Oppenheimer electronic energy differences. The situation with bond energies (BEs) is rather different, and their definition depends heavily depends on assumptions and theoretical frameworks.

As Cremer, Wu, Larsson, and Kraka have clearly put forward, [4] bond energies (BEs) are many times defined (and obtained) by assuming that the atomization energy ( $A E$ ) of a molecule, the sum of all its successive BDEs, is equal to the sum of the BEs of all of its bonds: $A E=\sum_{A} E_{0}^{A}-$ $\mathrm{E}($ molecule $)=\sum_{\mathrm{i}} \mathrm{BDE}_{\mathrm{i}}=\sum_{\mathrm{j}} \mathrm{BE}_{\mathrm{j}}$, $\mathrm{E}_{0}^{\mathrm{A}}$ being the ground state energy of isolated atom $\mathrm{A}$. Notice that a subtle extra assumption is made here. The second sum depends on an a priori list of bonds, whose origin is usually not disputed. This assumption is removed in the present approach, and BEs will be defined for all pairs of atoms (or fragments), whether they are chemically bonded or not. Actually, the BEs to be defined below allow for a direct classification of bonded and non-bonded interactions.

The AE used above implies a choice for the reference state of the atoms in which we atomize a molecule. By default, this is the ground state. However, if atomization into atoms as-in-the-molecule were possible, then the intrinsic atomization energy, IAE $=\sum_{A} E^{A}-E$ (molecule), where the sum adds the energy $E^{A}$ of all the atoms-in-the-molecule, would be equal to the sum of all the intrinsic bond energies $I A E=\sum_{j} I B E_{j}$. This defines the IBE as a direct descriptor of bond strength, since no reorganization effect other than the proper interaction of the already deformed atoms (or fragments, see below) to form the molecule is allowed.

IBEs are, in general, larger than BEs. This can be seen from the following identity,

$$
\sum_{i}\left(\mathrm{IBE}_{i}-\mathrm{BE}_{i}\right)=\mathrm{IAE}-\mathrm{AE}=\sum_{A}\left(E^{A}-E_{0}^{A}\right)=\sum_{A} E_{d e f}^{A}
$$

Here, $E_{\text {def }}^{A}$ is the deformation energy taking a ground state atom with energy $E_{0}^{A}$ into an atom-in-themolecule with energy $\mathrm{E}^{\mathrm{A}}$. This deformation includes all hybridization, promotion, charge transfer, electron delocalization, etc. energetic components previously commented, and is generally positive. This makes $\mathrm{IBE}_{\mathrm{i}}-\mathrm{BE}_{\mathrm{i}}>0$ in general. 


\section{Defining atoms (or fragments) in-the-molecule: the QTAIM and the IQA partition}

The biggest obstacle precluding a rigorous definition of an IBE is the (energetic) characterization of an atom or fragment in-the-molecule. Out of the many available proposals, the quantum theory of atoms in molecules devised by R. F. W. Bader and coworkers [24] is likely the best founded from the theoretical point of view. Regions in the physical space surrounded by local zero flux surfaces of the gradient field of the electron density, characterized by all points that comply with $\nabla \rho(\mathrm{r}) \cdot \mathrm{dn}=0$, where $\mathrm{n}$ is the normal vector to the interatomic surface at point $r$, are shown to be endowed with a large number of desirable properties.

The QTAIM provides an exhaustive partition of space, which is divided into regions that contain a single nucleus hence called quantum atoms. The few exceptions to this rule are also interesting themselves. [29] The atoms of the QTAIM may or may not share a common separating surface, the interatomic surface. In the first case these atoms are said to be "bonded", and a topological signature of this sharing, the so-called bond critical point, appears in the electron density. The interpretation of bond critical points has been a source of recent interesting debates, [30] but plays no role in the following. Clearly, the union of any number of QTAIM atoms defines a domain also surrounded by a zero flux surface, thus becoming a proper quantum region itself. In this way quantum fragments are introduced effortlessly.

Any properly defined one-electron operator density o $(r)$ may be integrated over a quantum region A to define an additive atomic expectation value: $\langle\mathrm{O}\rangle=\sum_{\mathrm{A}} \int_{\mathrm{A}} \mathrm{dr} \mathrm{o}(\mathrm{r})=\sum_{\mathrm{A}}\langle\mathrm{O}\rangle_{\mathrm{A}}$. Similarly, twoelectron operators give rise to pairwise additive domain expectation values:

$$
\langle G\rangle=\sum_{A, B} \int_{A} d r_{1} \int_{B} d r_{2} g\left(r_{1}, r_{2}\right)=\sum_{A, B}\langle G\rangle_{A, B} .
$$

Of utmost importace is the fact that QTAIM atoms provide unique domain-integrated kinetic energies for a large class of kinetic energy densities. [31,32] Notwithstanding the existence of an atomic virial theorem [33] that has been used to introduce additive atomic energies which recover the molecular energy at stationary points on potential energy surfaces, the above partition of one- and two-electron expectations leads to a general, exact decomposition of the energy, valid at any geometry. This is 
the IQA approach. $[25,26]$

Given a nuclear arrangement in the Born-Oppenheimer approximation, the total molecular energy under a Coulomb Hamiltonian depends only on $\rho\left(r_{1} ; r_{1}^{\prime}\right)$, the non-diagonal one-particle density matrix and on $\rho_{2}\left(r_{1}, r_{2}\right)$, the pair density, through $E=\operatorname{Tr}\left\{h \rho\left(r_{1} ; r_{1}^{\prime}\right)\right\}+(1 / 2) \operatorname{Tr}\left\{\rho_{2}\left(r_{1}, r_{2}\right) / r_{12}\right\}+$ $\sum_{A>B} Z_{A} Z_{B} / R_{A B}$, where $h=t-\sum_{A} Z_{A} / r_{A}$ is the one-electron hamiltonian. Separating all spatial integrations into quantum fragments, such that $R^{3}=\bigcup A$,

$$
\begin{aligned}
\mathrm{E} & =\sum_{\mathrm{A}} \int_{\mathrm{A}} \mathrm{h} \rho\left(\mathrm{r}_{1} ; \mathrm{r}_{1}^{\prime}\right) \mathrm{d} \mathrm{r}_{1}+\frac{1}{2} \sum_{\mathrm{A}, \mathrm{B}} \int_{\mathrm{A}} \mathrm{d} \mathrm{r}_{1} \int_{\mathrm{B}} \rho_{2}\left(\mathrm{r}_{1}, \mathrm{r}_{2}\right) / \mathrm{r}_{12}+\sum_{\mathrm{A}>\mathrm{B}} \frac{\mathrm{Z}_{\mathrm{A}} \mathrm{Z}_{\mathrm{B}}}{\mathrm{R}_{\mathrm{AB}}} \\
& =\sum_{\mathrm{A}}\left[\mathrm{T}^{\mathrm{A}}+\mathrm{V}_{\mathrm{ee}}^{\mathrm{AA}}+\mathrm{V}_{\mathrm{en}}^{\mathrm{AA}}\right]+\sum_{\mathrm{A}>\mathrm{B}}\left[\mathrm{V}_{\mathrm{en}}^{\mathrm{AB}}+\mathrm{V}_{\mathrm{ne}}^{\mathrm{AB}}+\mathrm{V}_{\mathrm{nn}}^{\mathrm{AB}}+\mathrm{V}_{\mathrm{ee}}^{\mathrm{AB}}\right] \\
& =\sum_{\mathrm{A}} \mathrm{E}_{\text {self }}^{\mathrm{A}}+\sum_{\mathrm{A}>\mathrm{B}} \mathrm{E}_{\mathrm{int}}^{\mathrm{AB}},
\end{aligned}
$$

All terms above are intuitive and chemically meaningful. The atomic (or fragment) self-energy, $\mathrm{E}_{\text {self }}^{\mathrm{A}}$, contains all the energetic terms pertaining to the group in vacuo: the kinetic energy of the electrons lying in the A domain, their inter-electron repulsion, and the Coulombic attraction to their nuclei. $E_{\text {self }}^{A}$ tends smoothly to $E_{0}^{A}$ if we adiabatically dissociate fragment $A$. Simply speaking, $E_{\text {self }}^{A}$ is the energy of a fragment in-the-molecule.

Similarly, $E_{i n t}^{A B}$ gathers all energy components mixing particles in two different regions: the repulsion of electrons (and nuclei) lying in A with those in $B$, and the attraction of electrons in one domain with the nuclei of the other. It is the interaction energy of the fragments-in-the-molecule. If fragments $A$ and $B$ are dissociated, $E_{i n t}^{A B}$ decays to zero, the leading term in the decay rate depending on the standard theory of intermolecular (inter-fragment) interactions. [34] As it has been stressed in many occassions, it is fruitful to separate the pair density into a classical term, depending on $\rho$, and a quantum-mechanical component, the exchange-correlation density: $\rho_{2}\left(r_{1}, r_{2}\right)=$ $\rho\left(r_{1}\right) \rho\left(r_{2}\right)-\rho_{x c}\left(r_{1}, r_{2}\right)$. This takes us to a parallel partition of $E_{i n t}^{A B}=E_{c l}^{A B}+E_{x c}^{A B}$, where the first term depends only on the classical electron and nuclear densities, and the second on the electronic exchange-correlation term. $E_{c l}^{A B}$ is a classical interaction. At large A,B distances it may be expanded via the multipolar approximation, and it is always dominated by the Coulombic interaction between the net charges born by $A$ and $B$. $E_{c l}^{A B}$ is thus the ionic component of the $A, B$ interaction. Since $\rho_{\mathrm{xc}}$ is intimately linked to the Fermi-Coulomb hole, [35] it is a measure of electron delocalization (or 
of electron population fluctuation, see below). $E_{x c}^{A B}$ measures the covalent component of the $A, B$ interaction.

\section{A real space in situ bond energy}

With these provisos, all the ingredients needed for a real space definition of an intrinsic bond energy, which we will call in situ bond energy, ISBE, are ready to be assembled.

Let us choose a molecule which we divide into fragments in whatever way we decide. The most resolved partition possible is atomic, and we will restrict ourselves to this in the following, without loss of generality. All our arguments are immediately generalized to a general fragmentation. Let us also choose in advance the reference energetic states of the isolated fragments (atoms), $\mathrm{E}_{0}^{\mathrm{A}}$. This choice plays no a priori role from a thermodynamic standpoint. Changing it simply resets the energy zero of each fragment. It may however play one if we are interested in following a given dissociation process adiabatically.

It is thus the case that the atomization energy is

$$
\begin{aligned}
-\mathrm{AE} & =\mathrm{E}-\sum_{\mathrm{A}} \mathrm{E}_{0}^{\mathrm{A}}=\sum_{\mathrm{A}}\left(\mathrm{E}_{\text {self }}^{\mathrm{A}}-\mathrm{E}_{0}^{\mathrm{A}}\right)+\sum_{\mathrm{A}>\mathrm{B}} \mathrm{E}_{\mathrm{int}}^{\mathrm{AB}} \\
& =\sum_{\mathrm{A}} \mathrm{E}_{\mathrm{def}}^{\mathrm{A}}+\sum_{\mathrm{A}>\mathrm{B}} \mathrm{E}_{\mathrm{int}}^{\mathrm{AB}},
\end{aligned}
$$

where, as before, all fragment (atomic) rearrangements upon molecule formation are absorbed in the deformation energy of the fragment, $E_{\text {def }}^{A}$.

This well-known expression $[25,26]$ allows us to understand why it is difficult to write the $A E$ as a sum of BEs. In the first place, all $A>B$ interactions have to be taken into account. Deciding in advance, for instance using chemical wisdom, which pairs of fragments (atoms) should or should not be included in the sum is risky. Freeing this assumption is easy. All pairs should be included in the sum. This leads to assigning bond energies to pairs of atoms which will not be traditionally bonded. Most times, these extra energies will be small. We think this is a fair price to pay for rigorousness. The second point hits the nucleus of the problem. If we insist on assigning BEs from the AE, we face how to divide the deformation energy of each fragment into pairwise additive contributions. This 
is, in principle, not possible. A fragment (atom) deforms in response to its interaction with all other fragments in the molecule. This is a true many body effect, that can only be considered as pairwise additive in an approximate way. In general, $\mathrm{E}_{\mathrm{def}}^{\mathrm{A}} \neq \sum_{\mathrm{B} \neq \mathrm{A}} \mathrm{E}_{\mathrm{def}}^{\mathrm{B}}$. Although work in this direction is clearly desirable, a direct BE definition is thus not straightforward.

The situation changes if we consider intrinsic bond energies instead. The fragments' reference state is that in-the-molecule, and

$$
\mathrm{IAE}=\sum_{\mathrm{A}} \mathrm{E}_{\mathrm{self}}^{\mathrm{A}}-\mathrm{E}=-\sum_{\mathrm{A}>\mathrm{B}} \mathrm{E}_{\mathrm{int}}^{\mathrm{AB}}
$$

Thus, If we are ready to pay the aforementioned price of releasing the assumption that only traditionally bonded fragments (atoms) contribute to IBEs, we can rigorously define an exactly pairwise additive intrinsic bond energy that we call in situ bond energy (ISBE) in the following:

$$
\operatorname{ISBE}_{\mathrm{AB}}=-\mathrm{E}_{\mathrm{int}}^{\mathrm{AB}}
$$

Notice that we abide to the common use of stabilizing ISBEs as positive quantities.

This sets up our protocol. To sum up, we have constructed a set of: (i) QTAIM atoms, which may be effortlessly gathered into fragments; (ii) a set of energies for these fragments (atoms) in-themolecule, $\mathrm{E}_{\text {self }}^{\mathrm{A}}$, which may be compared to previously chosen reference energies. These deformation energies of the fragments contain all relaxation effects leading to the in-the-molecule prepared state; (iii) a set of in situ bond energies for each pair of fragments. The ISBEs tell us about the energy released (in general) when the prepared fragments interact. Every ISBE has an ionic and a covalent component.

All these quantities depend only on the two Dirac observables $\rho$ and $\rho_{2}$. They are invariant under orbital transformations and may be either computed (as in the following) or experimentally determined. This last possibility is not straightforward, but accessible, for instance, through quantum crystallography techniques. [27]

An immediate link between the BDE of an $\mathrm{A}-\mathrm{B} \rightarrow \mathrm{A} \cdot+\mathrm{B}$. fragmentation process and the ISBE 
of the A-B link that is broken exists,

$$
\mathrm{BDE}=\mathrm{E}_{0}^{\mathrm{A}}+\mathrm{E}_{0}^{\mathrm{B}}-\mathrm{E}=\mathrm{ISBE}_{\mathrm{AB}}-\mathrm{E}_{\mathrm{def}}^{\mathrm{A}}-\mathrm{E}_{\mathrm{def}}^{\mathrm{B}} .
$$

Understanding the origin of deformation energies is important in order to connect standard BDEs to ISBEs. Fortunately, a number of real space techniques that shed light on this topic have been devised. A brief summary follows.

\section{Understanding the in-the-molecule state.}

In IQA, $E_{\text {def }}^{A}$ is the difference between the self-energies of two regions of space that contain the same number and type of nuclei. As such, the deformation energy may be analyzed in terms of the changes of the physical components of $\mathrm{E}_{\text {self }}^{\mathrm{A}}$ : its kinetic energy, electron-nuclear attraction and interelectron repulsion. This is useful, but seldom chemically appealing.

It has become common practice to separate the difference between the reference and the in-themolecule fragments into a component stemming from the change in the location of the nuclei, and a contribution from the modification in the number and distribution of the electrons therein contained, $E_{\text {def }}^{A}=E_{\text {def }}^{A, p r e p}+E_{\text {def }}^{A \text {,elec }}$. The first is a geometrical relaxation, usually called preparation, [36] that is normally taken into account via a standard two-step process in which we first distort adiabatically the reference geometry to that in-the-molecule and then we vertically allow for all electronic rearrangement in a second step: $E_{\text {def }}^{A, p r e p}=E_{0}^{A}(m o l)-E_{0}^{A}($ isol $)$. Here, mol and isol refer to the in-the-molecule and isolated geometries of the fragment, respectively. Usually, preparation energies are small, and not scrutinized further.

The electronic distortion, $\mathrm{E}_{\text {def }}^{\mathrm{A} \text { elec }}$, is more interesting, and contains all the terms previously commented: charge transfer $\left(E_{\text {def }}^{A, c t}\right)$, hybridization $\left(E_{\text {def }}^{A, \text { hyb }}\right)$, delocalization $\left(E_{\text {def }}^{A, \text { deloc }}\right)$, etc. One of the advantages of IQA is that, given a model wavefunction, we can perform an energetic decomposition and examine the effect of turning on or off this or that effect. In this way, access to hybridization energies, for instance, is possible. However, any of these procedures is neither orbital invariant nor independent of the method of calculation, and will not be pursued here anymore. We will restrict to invariant, in principle observable quantities. 
The charge transfer component of $E_{\text {def }}^{A, c t}$ is one of the contributions to $E_{\text {def }}^{A, e l e c}$ that admits such an invariant formulation. Using a grand canonical ensemble, [37] the thermodynamic energy cost (or energy release) upon extracting or adding a given number of electrons to a fragment is easily obtained. Let $\Delta N^{A}=N^{A}-N_{0}^{A}$ be the electron population change suffered by the reference fragment upon molecule formation. Here, $N^{A}=\int_{A} d r \rho(r)$ is the average number of electrons of $A$ in-the-molecule. If $\Delta N^{A}$ is negative we use the successive ionization potentials of $A, I P_{i}^{A}$. Let $\mathrm{n}=\operatorname{int}\left(\left|\Delta \mathrm{N}^{\mathrm{A}}\right|\right)$ and $\delta \mathrm{n}=\operatorname{frac}\left(\left|\Delta \mathrm{N}^{\mathrm{A}}\right|\right)$. Then $\mathrm{E}_{\text {def }}^{\mathrm{A}, \mathrm{ct}}=\sum_{\mathrm{i}}^{\mathrm{n}} \mathrm{IP}_{\mathrm{i}}^{\mathrm{A}}+\mathrm{IP}_{\mathrm{n}+1}^{\mathrm{A}} \delta \mathrm{n}$. If $\Delta \mathrm{N}^{\mathrm{A}}$ is positive we replace the ionization potentials with electron affinities (which may be zero). When $\Delta \mathrm{N}^{\mathrm{A}}$ is large and negative, i.e. if $A$ in the molecule is a cationic species, then $E_{\text {def }}^{A, c t}$ will be a considerably large quantity that will dominate $\mathrm{E}_{\mathrm{def}}^{\mathrm{A}}$.

Deep insight into the residual deformation component $E_{\text {def }}^{A, e c}-E_{\text {def }}^{A, c t}$ can be achieved by analyzing the electron distribution of the fragment in-the-molecule. A rigorous way to do that is via electron population distribution functions, EDFs. [38-40] The wavefunction of a molecule is not an eigenstate of the operator defining the number of electrons in fragment $A, \hat{N}_{A}$. This means that $N_{A}=\left\langle\hat{N}_{A}\right\rangle=$ $\int_{A} d r \rho(r)$, the average number of electrons in $A$ is not an eigenvalue of $\hat{N}_{A}$, so that measuring the number of electrons in the fragment will render values $\mathrm{n}_{\mathrm{A}}$ ranging from 0 to $\mathrm{N}$, the total number of electrons, with a defined set of probabilities, $p\left(n_{A}\right)$. This is the one-fragment EDF for fragment $A$. It is easy to show that the variance of this distribution is $\operatorname{var}\left(n_{A}\right)=N_{A}-\int_{A} d r_{1} \int_{A} d r_{2} \rho_{x c}\left(r_{1}, r_{2}\right)$. Multi-domain EDFs are also easily defined and if a partition of a molecule into $\mathrm{m}$ fragments is done, then $\mathrm{p}\left(\mathrm{n}_{\mathrm{A}}, \mathrm{n}_{\mathrm{B}}, \ldots, \mathrm{n}_{\mathrm{m}}\right)$, denote the probability of finding $\mathrm{n}_{\mathrm{A}}$ electrons in fragment $A$ and so on, where $\mathrm{n}_{\mathrm{A}}+\mathrm{n}_{\mathrm{B}}+\cdots+\mathrm{n}_{\mathrm{m}}=\mathrm{N}$. We call the set $\mathrm{S}=\left(\mathrm{n}_{\mathrm{A}}, \mathrm{n}_{\mathrm{B}}, \ldots, \mathrm{n}_{\mathrm{m}}\right)$ a real space resonance structure. These joint probabilities provide access to the full statistics of the electron population distribution, and are intimately linked to chemical bonding. For instance, multiple-center covariances are used to define multi-center bonding indices called n-center delocalization indices. [41-44] If electrons are spin-seggregated, then we come to spin-resolved EDFs, and a set of probabilities $\mathrm{p}\left(\mathrm{n}_{\mathrm{A}}^{\alpha}, \mathrm{n}_{\mathrm{A}}^{\beta}, \mathrm{n}_{\mathrm{B}}^{\alpha}, \mathrm{n}_{\mathrm{B}}^{\beta}, \ldots, \mathrm{n}_{\mathrm{m}}^{\alpha}, \mathrm{n}_{\mathrm{m}}^{\beta}\right)$ gives extremely fine-grained information about how electrons and their spins distribute. EDFs are defined in terms of observables, and may be determined again from quantum crystallography. If $S=\left(n_{A}^{\alpha}, n_{A}^{\beta}, n_{B}^{\alpha}, n_{B}^{\beta}, \ldots, n_{m}^{\alpha}, n_{m}^{\beta}\right)$ is a spin-resolved resonance structure, 
and just for the sake of completeness,

$$
\mathrm{p}(\mathrm{S})=\mathcal{P} \int_{\mathrm{D}} \Psi^{*} \Psi \mathrm{dx_{1 }} \mathrm{d} \mathrm{x}_{2} \cdots \mathrm{d} \mathrm{x}_{\mathrm{N}}
$$

where $\mathcal{P}=N ! /\left(n_{A}^{\alpha} ! \ldots n_{m}^{\alpha} ! n_{A}^{\beta} ! \ldots n_{m}^{\beta} !\right)$ is an indistinguishability factor, and $D$ a domain in which the first $n_{A}^{\alpha} \alpha$ electrons are integrated over fragment $A$, etc, and the last $n_{m}^{\beta} \beta$ electrons over fragment $m$. In this expression, $x_{i}$ is a full electron coordinate consisting of both spin and spatial parts.

This short description does not exhaust the battery of real space methods that can be used to get information about the origin of deformations, but reasonably summarizes the basic toolkit at our disposal. For instance, a partition of the bond order into one-electron contributions, can be obtained through the use of natural adaptive orbitals (NAdOs). [45] A brief discussion is found in the Electronic Supporting Information.

\section{Using the protocol}

We now turn to examine a few systems that exemplify several of our claims. We study the dissociation of $\mathrm{LiF}$ and $\mathrm{LiH}$ at the Coupled Cluster with Single and Double excitations level with the aug-cc-pVDZ one-electron basis set (CCSD/aug-cc-pVDZ), which provides reasonable ionization potentials and electron affinities for all the atoms in vacuo. These calculations have been performed using the pySCF suite. [46] Then we follow two atomization processes with size-consistent valence Complete Active Space Self Consistent Field (CASSCF) calculations with Def2-TZVPP basis set, performed with a locally modified version of GamessUS [47]. We examine the full atomization of methane $\mathrm{CH}_{4}\left({ }^{1} \Sigma_{g}\right) \longrightarrow \mathrm{C}\left({ }^{3} \mathrm{P}\right)+4 \mathrm{H}\left({ }^{1} \mathrm{~S}\right)$ by means of a scaling procedure that preserves the $\mathrm{T}_{\mathrm{d}}$ symmetry in which all the $\mathrm{C}-\mathrm{H}$ bond lengths are simultaneously scaled towards dissociation. Finally the dissociation of dinitrogen $\mathrm{N}_{2}\left({ }^{1} \Sigma_{g}\right) \longrightarrow 2 \mathrm{~N}\left({ }^{4} \mathrm{P}\right)$, is analyzed. The CASSCF level used in the last two processes was preferred in place of the CCSD or multi-reference configuration interaction $(\mathrm{MRCl})$ methods because the latter, albeit more accurate and including dynamical correlation, are not immediately size-consistent. The use of the CASSCF method, which can be easily taylored to enjoy this property, guarantees the correct dissociation of both systems into their fragments, as well as reasonable values of the deformation energies during the whole potential energy curves. On the 
Table 1: LiF and LiH (AX) ISBE data. CCSD(valence)/aug-cc-pVTZ calculation. Net charges in a.u., all other energetic data in $\mathrm{kcal} / \mathrm{mol}$

\begin{tabular}{lrrrrrrrr}
\hline System & $\mathrm{Q}_{\mathrm{A}}$ & $\mathrm{D}_{\mathrm{e}}=\mathrm{BDE}$ & $\mathrm{E}_{\text {def }}^{\mathrm{A}}$ & $\mathrm{E}_{\text {def }}^{\mathrm{X}}$ & $\mathrm{E}_{\text {int }}^{\mathrm{AX}}=-1 \mathrm{ISBE}$ & $\mathrm{E}_{\mathrm{cl}}^{\mathrm{AX}}$ & $\mathrm{E}_{\mathrm{xc}}^{\mathrm{AX}}$ & $\mathrm{E}_{\text {def }}+\mathrm{E}_{\mathrm{cl}}^{\mathrm{AX}}$ \\
\hline $\mathrm{LiF}$ & 0.934 & -131.7 & 132.1 & -55.7 & -208.1 & -179.3 & -28.8 & -102.9 \\
$\mathrm{LiH}$ & 0.906 & -56.8 & 121.0 & 4.9 & -182.6 & -158.7 & -23.9 & -32.8 \\
\hline
\end{tabular}

other hand, the IQA method requires well-defined one-particle, $\rho\left(r_{1} ; r_{1}^{\prime}\right)$, and two-particle, $\rho_{2}\left(r_{1}, r_{2}\right)$, densities, so that the CASPT2 level, has not been used either.

QTAIM domains and IQA analyses have been performed with our Promolden [48] code. Almost all systems presented difficulties with geometries in the repulsive region either because a group was ionized (carbon in methane) or because a non-nuclear maximum appeared. These are not considered in this work. Numerical integration grids were chosen to guarantee reconstruction of total molecular energies to an accuracy better or equal than $1 \mathrm{kcal} / \mathrm{mol}$. Finally EDFs and NAdO's where obtained from the Promolden atomic overlap matrices with the EDF [49] code.

\section{Choosing a reference: The LiF and LiH cases}

We first show how the protocol provides valuable information about reference states in two classical examples, the LiF and $\mathrm{LiH}$ diatomics. Both are usually classified as heavily ionic, but non-orthogonal valence bond [50-52] as well as energy decomposition [36] (EDA) analyses with neutral fragmentation tend to classify $\mathrm{LiH}$ as a rather covalent system.

Table 1 contains a summary of our computed results. At this level of theory, both the ionization potential (IP) of $\mathrm{Li}$ and the electron affinities $(\mathrm{AE})$ of $\mathrm{F}$ and $\mathrm{H}$ are reasonably represented, being equal to $123.2,-80.4$ and $-16.8 \mathrm{kcal} / \mathrm{mol}$, in that order, which are to be compared with the experimental values, $124.3,-78.4$, and $-17.4 \mathrm{kcal} / \mathrm{mol}$, respectively. The first interesting point lies in the QTAIM net charges, very close to the nominal ionic values. As expected from electronegativity arguments, charge transfer in LiF is slightly larger than in $\mathrm{LiH}$, but the value in the latter system is still very large. This points to large ionic components in the ISBEs.

Let us turn to the BDE, which is $75 \mathrm{kcal} / \mathrm{mol}$ larger in $\mathrm{LiF}$ than in $\mathrm{LiH}$. Our ISBEs are larger than the BDEs (as expected), with a considerable smaller difference between them, 208 and $183 \mathrm{kcal} / \mathrm{mol}$, just $25 \mathrm{kcal} / \mathrm{mol}$ in favor of LiF. This immediately tells us that the low BDE of $\mathrm{LiH}$ is not due to the intrinsic strength of the link. Actually, out of the -208.1 and $-182.6 \mathrm{kcal} / \mathrm{mol} \mathrm{E}_{\text {int }}^{\mathrm{AB}}$ values in $\mathrm{LiF}$ and 
$\mathrm{LiH}$, respectively, a very large part, -179.3 and -158.7 , respectively, comes from the $\mathrm{E}_{\mathrm{cl}}$ component. This amounts to $86 \%$ in both cases, close to the Madelung contribution to lattice energies in ionic crystals. [53] Moreover, the $Q_{A} Q_{B} / R_{A B}$ monopole-monopole term is immediately computed, equal to -184.5 and $-170.9 \mathrm{kcal} / \mathrm{mol}$ for $\mathrm{LiF}$ and $\mathrm{LiH}$. This implies that higher order multipolar terms, i.e. larger polarization in the density distribution, play a significant role in the electrostatics of $\mathrm{LiH}$. A multipole analysis (directly obtained from the QTAIM analysis) shows that the electronic dipole moments of the $\mathrm{Li}$ and $\mathrm{H}$ quantum atoms are -0.05 and -0.42 a.u., respectively. The distribution of electrons in both atoms polarizes toward the $\mathrm{H} \rightarrow \mathrm{Li}$ direction (this is nothing but the well known large forward polarization of anions and the small response-like backward polarization of cations), but the effect is 8 times more intense in $\mathrm{H}$. Of course, a similar effect is found in LiF, but the Li, F dipoles are smaller ( -0.02 and -0.25 a.u., respectively). According to this clear analysis, the intrinsic bond in both systems is largely ionic. On top of this, a small, yet non-negligible covalent contribution to the $E_{i n t}=-I S B E$ exists. It is similar in both systems, -29 and $-24 \mathrm{kcal} / \mathrm{mol}$. Its role will be commented below.

As the ISBEs show, the links in $\mathrm{LiF}$ and $\mathrm{LiH}$ are considerably strong, but the $\mathrm{BDE}$ in $\mathrm{LiH}$ is rather small. Given our previous comments, the fragments' deformations are in charge of this difference. As expected from our physical arguments, large charge transfers are followed by large $\mathrm{E}_{\mathrm{def}}^{\mathrm{ct}}$ components. Using our computed IPs and AEs together with the fragments' net charges, the partial ionization of the Li moiety should cost 115.1 and $111.6 \mathrm{kcal} / \mathrm{mol}$ in $\mathrm{LiF}$ and $\mathrm{LiH}$, respectively. Similarly, the energy release due to the formation of the anions should equal -75.1 and $-15.2 \mathrm{kcal} / \mathrm{mol}$. Table 1 shows that the $\mathrm{E}_{\mathrm{def}}^{\mathrm{Li}}$ deformations are only 17.0 and $9.4 \mathrm{kcal} / \mathrm{mol}$ higher than these grand canonical values. The Li cations have suffered an almost pure (partial) ionization, with small extra electron reorganization. The latter is a bit larger in LiF, in agreement with the more polarizing character of the $\mathrm{F}^{-}$anion. Similar arguments apply to the formation of the anionic species. In LiF, $\mathrm{E}_{\text {def }}^{\mathrm{F}}=-55.7 \mathrm{kcal} / \mathrm{mol}$ (to be compared with -75.1 ), and all the extra electron reorganization cost is hence equal to $19.4 \mathrm{kcal} / \mathrm{mol}$. In $\mathrm{LiH}$, with $\mathrm{E}_{\text {def }}^{\mathrm{H}}=+4.9 \mathrm{kcal} / \mathrm{mol}$, the non charge-transfer costs amount to $20.1 \mathrm{kcal} / \mathrm{mol}$. The consistency of these numbers is rather interesting, in our opinion.

The role of the choice of reference states is now clear. If we use the BDE with respect to neutral ground state fragments, the Li moiety in-the-molecules lies between 121 and $132 \mathrm{kcal} / \mathrm{mol}$ above the in vacuo ${ }^{2} \mathrm{~S}$ - Li atom. These numbers are -56 and $+5 \mathrm{kcal} / \mathrm{mol}$ for $\mathrm{F}$ and $\mathrm{H}$, respectively. However, 
if we turn to ionic reference states (closed shell ${ }^{1} \mathrm{~S} \mathrm{Li}^{+}, \mathrm{F}^{-}$, and $\mathrm{H}^{-}$), then the in-the-molecule $\mathrm{Li}^{+}$ cations lie just 8.9 and $-2.2 \mathrm{kcal} / \mathrm{mol}$ above the reference, respectively, and the $\mathrm{F}^{-}$, and $\mathrm{H}^{-}$anions 24.7 and $21.7 \mathrm{kcal} / \mathrm{mol}$, respectively. Although a matter of taste from the thermodynamic point of view, the ionic reference state is clearly to be preferred, the total deformation with respect to it being 33.7 and $19.5 \mathrm{kcal} / \mathrm{mol}$ in $\mathrm{LiF}$ and $\mathrm{LiH}$.

What is the reason, then, behind the very different BDEs in these two systems? It has been previously shown [54] that in heavily charged systems the total ionization energy cost, the energy loss due to the formation of (partial) cations and anions plus the electrostatic energy gain due to Coulombic attraction of the charged species, provides interesting clues to bonding. A system's tendency toward charge transfer is due to a total stabilizing value for this quantity, that may be evaluated rigorously through $E_{\text {def }}^{A, c t}$. In Table 1 we simply show $E_{d e f}+E_{c l}$. The gain due to ionization is about $70 \mathrm{kcal} / \mathrm{mol}$ larger in $\mathrm{LiF}$ than in $\mathrm{LiH}$. In the latter, this gain is just $-32.8 \mathrm{kcal} / \mathrm{mol}$, competing clearly with the covalent contribution, equal to $-23.9 \mathrm{kcal} / \mathrm{mol}$. This is the reason why valence bond calculations and neutral EDA decompositions provide a largely covalent image of $\mathrm{LiH}$. And behind all this, one can simply recognize that the difference in EAs between $\mathrm{F}$ and $\mathrm{H}$ is about $64 \mathrm{kcal} / \mathrm{mol}$ (calculated values), very close to the difference in ionization gains. The $75 \mathrm{kcal} / \mathrm{mol}$ difference in $\mathrm{BDEs}$ is, in the end, largely dominated by the difference in the $\mathrm{F}$ and $\mathrm{H}$ electron affinities. With our numbers, it is due to different ionization total costs $(70 \mathrm{kcal} / \mathrm{mol})$ plus 5 extra $\mathrm{kcal} / \mathrm{mol}$ coming from covalency. We firmly believe that this simple analysis shows how ISBEs and deformation energies may shed light in chemical bonding issues.

\section{A case with traditional atomic promotion: the atomization of methane}

The full atomization (or formation) of methane, $\mathrm{CH}_{4} \rightarrow \mathrm{C}\left({ }^{3} \mathrm{P}\right)+4 \mathrm{H}\left({ }^{2} \mathrm{~S}\right)$ has served several times as a prototype to show the need of IBEs. With two unpaired electrons, the triplet ground state of the carbon atom is naïvely inconsistent with the four bonding Lewis pairs existing in methane. It is thus assumed that a large preparation energy should be involved in the symmetry preserving formation of $\mathrm{CH}_{4}$ from four ground state hydrogens. Besides polarization and (small) charge transfer efffects, chemists expect that most of the preparation energy be due to: (i) excitation from the ${ }^{3} \mathrm{P}$ to the ${ }^{5} \mathrm{~S}$ excited state, the latter with four unpaired electrons; (ii) hybridization of the quintet to four equivalent 
Table 2: $\mathrm{CH}_{4}$ data at the CAS[8,8]//Def2-TZVPP equilibrium geometry. Net charges in a.u., all other energetic data in $\mathrm{kcal} / \mathrm{mol}$

\begin{tabular}{rrrr}
\hline & $Q_{C}$ & $E_{\text {def }}^{C}$ & $E_{\text {def }}^{H}$ \\
\hline \hline$E_{\text {int }}^{\mathrm{CH}}$ & 0.026 & 62.2 & 27.1 \\
\hline-128.3 & $E_{\mathrm{C}}^{\mathrm{CH}}$ & $\mathrm{E}_{\mathrm{int}}^{\mathrm{HH}}$ & $\mathrm{E}_{\mathrm{xc}}^{\mathrm{HH}}$ \\
\hline
\end{tabular}

$\mathrm{sp}^{3}$-like bonding functions.

Assuming four identical $\mathrm{C}-\mathrm{H}$ bonds (and exclusively four bonds), the sequential sum of the four BDEs provides a thermodynamic BE of about $104 \mathrm{kcal} / \mathrm{mol}$. [4] The triplet to quintet excitation energy is obviously well known, $96.5 \mathrm{kcal} / \mathrm{mol}$, [55] and there are several estimations of the hybridization costs. Since the latter are clearly approximations, we will take one of the published possibilities, $[56,57] 62 \mathrm{kcal} / \mathrm{mol}$. Ignoring all other factors, the deformation of $\mathrm{C}$ turns out to be about 159 $\mathrm{kcal} / \mathrm{mol}$. If, as usually done, the $\mathrm{H}$ atom is expected to bind without further modifications (see below, however) then, as already noticed, [4] the $\mathrm{C}-\mathrm{H}$ IBE should scale to about $104+159 / 4 \approx 144$ $\mathrm{kcal} / \mathrm{mol}$, a significantly higher value than the plain $\mathrm{BE}$.

Our CAS data provides an equilibrium C-H distance equal to $1.101 \AA$, with rather neutral fragments. The net charge of $\mathrm{C}$ is 0.026 a.u., with basically neutral hydrogen atoms bearing -6.5 me. Charge transfers are thus small, so we may concentrate on electron reorganizations. The $\mathrm{BE}_{\mathrm{CH}}$ obtained via $\mathrm{AE}=4 \mathrm{BE}_{\mathrm{CH}}$ is reasonable, $94 \mathrm{kcal} / \mathrm{mol}$. Since we want to explore the changes along the dissociation process, thus needing a size-consistent technique like valence CAS, we believe that this accuracy fits our needs.

The basic data are reported in Table 2. The in situ bond energy of the $\mathrm{C}-\mathrm{H}$ link, $128.3 \mathrm{kcal} / \mathrm{mol}$, is $34 \mathrm{kcal} / \mathrm{mol}$ larger than the calculated standard BE. This difference is just $10 \mathrm{kcal} / \mathrm{mol}$ lower than the one obtained from the abovementioned approximations. As well-known from IQA, in this case the components of $E_{i n t}$ are completely different than in $\mathrm{LiF}$ or $\mathrm{LiH}$. The $\mathrm{E}_{\mathrm{int}}=-\mathrm{ISBE}$ is dominated by covalency, with a destabilizing $\mathrm{E}_{\mathrm{cl}}=21.2 \mathrm{kcal} / \mathrm{mol}$ term due to the electrostatic repulsion of the two basically neutral $\mathrm{C}$ and $\mathrm{H}$ species. As anticipated, the $\mathrm{H}-\mathrm{H}$ interaction is small, it is a secondary interaction, [58] but cannot be neglected. Each of the $6 \mathrm{H}-\mathrm{H}$ pairs has a exchange-correlation driven ISBE of about $5 \mathrm{kcal} / \mathrm{mol}$. We have argued that these non-bonded terms are the short-range remnants of long-range dispersion. [59] If we do not recognize these 1,3 secondary interactions and insist on asigning this stabilization to the chemical $\mathrm{C}-\mathrm{H}$ bonds, then each $\mathrm{C}-\mathrm{H}$ bond energy would 
acquire an extra $(6 \times 4.8) / 4=7.2 \mathrm{kcal} / \mathrm{mol}$ stabilization energy. We will not comment further on this topic in this introductory paper, but we mention that imbalance among these 1,3 interactions lies behind systematic variations of tabulated $\mathrm{C}-\mathrm{H}$ bond energies on passing from $\mathrm{CH}_{4}$ to $\mathrm{CH}_{3}-,-\mathrm{CH}_{2}-$, etc, that deserve further study.

FIG.1

The behavior of deformation energies with respect to ground state atoms is shown in Fig. 1. Both $\mathrm{E}_{\text {def }}$ 's decay to zero at large $\mathrm{C}-\mathrm{H}$ distance, and rise abruptly, dominated by the increase of the atomic kinetic energies, at short distance. $E_{\text {def }}^{C}$ as well as $E_{\text {def }}^{H}$ show a maximum, inflection, minimum sequence as we decrease the $\mathrm{C}-\mathrm{H}$ distance at distances larger than but relatively close to equilibrium. This behavior has been repeatedly found in dissociation curves, and its meaning will be further discussed. It provides a relatively wide $\mathrm{C}-\mathrm{H}$ distance region in which $\mathrm{E}_{\text {def }}$ is relatively constant. As expected, the relaxation of the $\mathrm{C}$ atom, $62.2 \mathrm{kcal} / \mathrm{mol}$, is larger than that of $\mathrm{H}, 27.1$ $\mathrm{kcal} / \mathrm{mol}$, but the latter is not negligible, as assumed in naïve treatments. On the contrary, the relaxation of the four $\mathrm{H}$ atoms, $108.4 \mathrm{kcal} / \mathrm{mol}$ dominates the total deformation of the system, 170.6 $\mathrm{kcal} / \mathrm{mol}$. Interestingly, this quantity is similar to the sum of the triplet to quintet excitation plus the estimated hybridization cost just commented. This helps in understanding the similarity of the two intrinsic bond energies.

A detailed picture of the promotion process from isolated atoms to the in-the-molecule state can be followed through the EDF's. For the sake of conciseness, Fig. 2 shows the evolution of the spinless two-fragment EDF. $p(S)$ is the probability of each reasonance structure $S=\left(n_{C}, n_{4 H}\right)$, i.e. the probability of finding $\mathrm{n}_{\mathrm{C}}$ electrons in the $\mathrm{C}$ atom and the rest in the four equivalent hydrogens. Several relevant points deserve comment.

\section{FIG.2}

Firstly, the probability of the neutral resonance structure, with 6 electrons in the $C$ atom, tends obviously to one as we approach the dissociation limit. At equilibrium, however, this value has decreased to about 0.32 . This has strong implications about the meaning of promotion and/or hybridization energies for atoms-in-the-molecules. Delocalization (covalency) effects are important in such a covalent system, and most times we count the number of electrons in the $\mathrm{C}$ atom in methane, we do not find 6 . The analysis may be extended significantly. The first process to set on as we approach the $\mathrm{H}$ atoms is that of one electron exchange, followed by two-, three-, and four- 
electron exchanges. All of these exchange processes are basically symmetric (pure covalency) up to about $2.2 \AA$. From this distance upt to close to equilibrium the carbon moiety is substantially more electronegative than the $\mathrm{H}$ atoms, and negatively charged carbon resonance structures dominate. However, as we approach equilibrium this effective electronegativity difference decreases, and the equilibrium net charges are small. Notice that past the equilibrium geometry the sign of charge transfer is clearly inverted, with negatively charged hydrogens. We remark that the plateaus of $E_{\text {def }}$ correlate with the EDF behavior.

\section{FIG.3}

More insight into the $\mathrm{C}$ excitations can be obtained from the neutral $S=(6,4)$ structures. Spinresolving these (and only these) contributions, we get Fig. 3a, where the normalized to one $\mathrm{p}(\mathrm{S})$ of the $(6,4)$ spinless structure are plotted. The quasi- $M_{S}$ quantum number of the $C$ atom is obtained as half the difference between the number of $\alpha$ and $\beta$ electrons, $\left(n_{C}^{\alpha}-n_{C}^{\beta}\right) / 2$. At large distances, the 6-electron $\mathrm{C}$ atom is in its ${ }^{3} \mathrm{P}$ ground state, so the probability of each of its the three $\mathrm{M}_{\mathrm{S}}$ values, $M_{S}=0, \pm 1$ is equal to $1 / 3$. No other contribution arises. As we decrease the distance and bonding mechanisms start to take place, the $\mathrm{M}_{\mathrm{S}}= \pm 1$ components are quenched. Notice that in the very short-range distance regime the probabilities have turned basically binomial, due to quasi-independent electrons, with $\mathrm{p}\left(\mathrm{M}_{\mathrm{S}}=0\right) \approx 0.5$ and $\mathrm{p}\left(\mathrm{M}_{\mathrm{S}}= \pm 1\right) \approx 1 / 4$. As basically pure covalent bonds are formed, electrons lose memory of their previous state when they were part of the fragments. We will use this terminology a few more times to indicate how much the original electron distribution, e.g. the spin structure, of the fragments is maintained in the final molecule. This memory loss behavior has already been put forward. [40] More interesting is the appearance of a $\mathrm{M}_{\mathrm{S}}= \pm 2$ component at intermediate distances, which peaks at about a C-H distance of $1.8 \AA$. This is not found at dissociation, and points to the sought spin excitation.

If we assume that the 6-electron carbon atoms do only undertake minimal inter-configurational rearrangements, then we are led to interpret the normalized spin-resolved EDFs in terms of the ${ }^{3} \mathrm{P},{ }^{1} \mathrm{~S}$, and ${ }^{1} \mathrm{D}$ multiplets emerging from the ground state $2 \mathrm{~s}^{2} 2 \mathrm{p}^{2}$ configuration and the ${ }^{5} \mathrm{~S}$ from the $2 s^{1} 2 p^{3}$ one to allow for $M_{S}= \pm 2$. Using a ladder-like argument, all $M_{S}= \pm 2$ states come from promotions to the ${ }^{5} \mathrm{~S}$. Deleting the appropriate number of $\mathrm{M}_{\mathrm{S}}= \pm 2, \pm 1,0$ states, all remaining $\mathrm{M}_{\mathrm{S}}= \pm 1$ states should come from the ${ }^{3} \mathrm{P}$. Repeating this process, all the rest $\mathrm{M}_{\mathrm{S}}=0$ states will correspond to the singlets, which we cannot resolve further. This leads to Fig. 3b, where the proportion of the 
different multiplets is followed along dissociation. As the $\mathrm{C}$ atom binds to the hydrogens, we may imagine that all possible intra-configurational multiplets become accessible. What our calculations show is that the interconfigurational ${ }^{5} \mathrm{~S}$ is actually populated, with a maximum contribution close to $20 \%$ in the region where $E_{\text {def }}^{C}$ peaks, but that it is relatively unimportant at equilibrium. The deformation energy of the $\mathrm{C}$ atom in methane is then not dominated by the ${ }^{3} \mathrm{P}$ to ${ }^{5} \mathrm{~S}$ excitation, although this may play a role during bond formation. At all distances, the ${ }^{3} \mathrm{P}$ multiplet dominates the spin structure of the neutral resonance structure. This memory effect has also been previously reported. [60] We also stress that possible correlations between the change in atomic electronegativity and the population of excited intra-configurational states may exist, deserving further studies. As in other cases, the appearance of a maximum in the deformation energy of the interacting atoms as they bind may be tracked down to changes in the fragments' electronic structure. These maxima tend to coincide with the inflection points of the dissociating resonance structures, $p(6,4)$ in the case of methane, as Fig. 2 testifies.

Finally, we notice that many of the assumptions of current models to obtain bond energies are not supported by rigourous theoretical analyses like the ones herein presented. Secondary interactions will play a significant role in many interesting cases, and delocalization effects change the instantaneous electron count of atoms-in-the-molecules so as to render traditional promotion ideas almost useless. An atom-in-a-molecule is, after all, a complex object.

\section{Deformation in the absence of traditional atomic promotion: the dissociation of $\mathbf{N}_{2}$}

The dissociation of dinitrogen, $\mathrm{N}_{2} \rightarrow 2 \mathrm{~N}\left({ }^{4} \mathrm{~S}\right)$, offers an opportunity to consider a system which needs no classical promotion of its constitutent atoms to another spin state to form the triply bound diatomic. Moreover, if we are satisfied with the canonical $\sigma-\pi$ bonding picture and do not invoke banana bonds, for instance, then the $2 \mathrm{p}^{3}$ electrons need not re-hybridize either. In a first approximation, the relaxation energy of the $\mathrm{N}$ atoms lack the basic traditional components and may be taken as vanishing.

Our computations, Table 3, provide a large ISBE of about $290.7 \mathrm{kcal} / \mathrm{mol}$, dominated by its covalent component, $E_{x c}=-429 \mathrm{kcal} / \mathrm{mol}$. We have noticed in the past [61] that it is $E_{x c}$, the covalent 
Table 3: $\mathrm{N}_{2}$ data at the CAS[10,8]//Def2-TZVPP equilibrium geometry, $\mathrm{d}(\mathrm{N}-\mathrm{N})=1.104 \AA$. Energies in $\mathrm{kcal} / \mathrm{mol}$

\begin{tabular}{rrrrr}
\hline $\mathrm{D}_{\mathrm{e}}$ & $\mathrm{E}_{\text {def }}^{\mathrm{N}}$ & $\mathrm{E}_{\text {int }}^{\mathrm{NN}}$ & $\mathrm{E}_{\mathrm{cl}}^{\mathrm{NN}}$ & $\mathrm{E}_{\mathrm{ic}}^{\mathrm{NN}}$ \\
\hline 212.0 & 39.2 & -290.7 & 138.4 & -429.1
\end{tabular}

contribution to the interaction energy, that scales linearly with bond order, in agreement with standard wisdom. It has now been proven, $[62,63]$ that the standard measure of covalent bond order in real space, the delocalization index, is indeed rigorously related to $E_{x c}$, putting firm theoretical ground to the usual bond-energy bond-order correlations. [64] We also remark that the small equilibrium distance lies behind the important $E_{c l}$ electrostatic destabilization energy that significantly lowers $\mathrm{E}_{\mathrm{xc}}$ to the final ISBE.

The in-the-molecule state of the $\mathrm{N}$ atom lies $39.2 \mathrm{kcal} / \mathrm{mol}$ above the ${ }^{4} \mathrm{~S}$ ground state. This is smaller than the $62.2 \mathrm{kcal} / \mathrm{mol}$ of $\mathrm{C}$ in methane, but definitely not zero. So, indeed, the $\mathrm{N}$ atom deforms less than $\mathrm{C}$, as expected, but the total deformation of the system, $78.4 \mathrm{kcal} / \mathrm{mol}$, is not negligible. The evolution of $\mathrm{E}_{\mathrm{def}}^{\mathrm{N}}$ with the $\mathrm{N}-\mathrm{N}$ distance shows again, as in methane, a maximum, inflection, mimimum sequence providing a plateau that extends from about equilibrium to $1.6 \AA$.

FIG.4

Fig. 4 shows how the behavior uncovered in $\mathrm{CH}_{4}$ is rather common. The neutral $\mathrm{S}=(7,7)$ resonance structure decays sigmoidally from $\mathrm{p}(\mathrm{S})=1$ at dissociation to the binomial behavior at short distance. Again, considering populationally undistorted $\mathrm{N}$ atoms at the equilibrium geometry is a very poor approximation. There is only $40 \%$ probability that we measure $n=7$. As we progressively decrease the distance from equilibrium, first the one-electron exchanges (this time necessarily symmetric) set in, and at a later stage, the two- and three-electron exchanges appear.

FIG.5

Focusing on the 7-electron nitrogens, Fig. 5a displays at dissociation an equiprobable normalized spin-resolved EDF distribution of the four $M_{S}= \pm 3 / 2, \pm 1 / 2$ components of the quartet, with $\mathrm{p}=1 / 4$, together with spin-quenching as bonding occurs. However, this time no extra $\mathrm{M}_{\mathrm{S}}$ spin component is visible during the process, and no interconfigurational excitation is needed to account for the computed data. In this sense, the original quartet suffices to understand bonding, in relative agreement with intuition. If we interpret again the changes in terms of the three intra-configurational multiplets of the $2 \mathrm{p}^{3}$ configuration, Fig. $5 \mathrm{~b}$ shows an exchange of the roles of the high-spin ${ }^{4} S$ and 
the low-spin ${ }^{2} \mathrm{D}+{ }^{2} \mathrm{P}$ doublets. In contrast to methane, at equilibrium the 7 electron resonance structures of the $\mathrm{N}$ atom do not keep much memory of their original state, and the highest populated multiplets are spin-quenched doublets. The crossing between the quartet and the doublet occurs at about the distance at which the $E_{\text {def }}$ curve shows its maximum. All this shows neatly the subtleties behind the final values of relaxation or promotion energies.

Summarizing, the dinitrogen example shows that even in the most simple possible cases we should expect non-trivial phenomena accounting for the deformation of the interacting fragments. As we have tried to show, most of the assumptions currently in use (constancy of the fragments' electron count, simple multiplet excitations to allow for the correct number unpaired electron, hybridization, etc) do not hold or, in the best case, are to be understood as very crude approximations. We think that the protocols here reported may help improve this situation.

\section{Conclusions}

We have shown in this work how the use of real space fragments emanating from the quantum theory of atoms in molecules [24] (QTAIM) leads to a consistent definition of intrinsic bond strengths, the so-called in situ bond energies (ISBEs). These quantities comply with all the standard thermochemical requisites to be used as rigorous energetic indicators of bond strength. We argue that, in line with recent proposals, [18] the global energetic standpoint regarding bond strength should not be abandoned in favor of local measures like force constants. However, and this time in agreement with the supporters of local views, [4] we also show that bond dissociation energies (BDEs) are indeed difficult to reconcile with rigor with individual bond energies. These difficulties may all be solved if the interaction strength between two fragments $A$ and $B$ is measured from the energies of these fragments in-the-molecule. In a fragmentation process $A-B \rightarrow A \cdot+B \cdot$ the $B D E$ is then equal to the sum of the energy costs to deform the isolated fragments into their in-the-molecule state, the fragment' deformation energies, and an intrinsic bond energy. Over the years, the definition of intrinsic bond energies has suffered from a severe lack of rigor in accessing proper in-the-molecule energies. The use of real space tools successfully tackles all these questions at a time. Given a decomposition of a molecule into QTAIM fragments, which can smoothly vary from an atomic partition to a coarse-grained division into just two-fragments, the interacting quantum atoms approach $[25,26]$ 
(IQA) permits an exact decomposition of the total molecular energy into a sum of self-energies $E_{\text {self }}^{A}$ for each of the fragments $A$ and a set of pairwise additive interaction energies among them, $E_{\text {int }}^{A B}$. If the total atomization (or fragmentation, in general) energy is examined, this is then exactly equal to the sum of the atomic (or fragment) deformation energies with respect to their energies at dissociation, and their mutual interactions, or in situ bond energies. This exact result comes at the price of introducing ISBEs for each pair of fragments, be them chemically bonded or not. We firmly think that considering bond energies only for traditionally bonded pairs is a practice that may lead to considerable errors. Using standard IQA practice, $[25,26]$ the ISBEs can be further divided into ionic and covalent components, so that a chemically appealing picture of bond energy descriptors appear.

All the energetic quantities in the protocol herein introduced are observable, amenable in principle to experimental determination. We nevertheless acknowledge that the electronic pair density is still not easily accessible from standard techniques, although we also argue that advances in the field of quantum crystallography may soon change this. [27]

Deformation energies build the bridge between BDEs and ISBEs. They hold all the clues about how far the fragments are from the reference states we decide to use. In the present protocol, changing the reference state of the fragments does only reset the zero of self-energy, affecting no other quantity. Moreover, many real space tools may be used to gain insight about the origin of deformations, which can again be rigourously partitioned into charge transfer and purely electronic components. We have used here, as an example, electron number distribution functions, $[39,40]$ (EDFs) which provide a meaningful picture of how the electrons in a fragment are organized, offering valuable information about the in-the-molecule electronic state of a fragment.

To demonstrate the power of the protocol, we have examined three prototype examples. The LiF and $\mathrm{LiH}$ molecules show how to rationalize two very different BDEs that share rather similar ISBEs. We show, beyond doubt, that the state of the atoms-in-the-molecules is considerably closer to the ionic reference than to the atomic one, and we illustrate how the IQA numbers immediately lead to identifying the difference in the electron affinities of the $\mathrm{F}$ and $\mathrm{H}$ atoms as the driving force behind the different BDEs. Two other atomization processes, the dissociation of methane into its ground state $\mathrm{C}$ and $\mathrm{H}$ atoms and the standard dissociation of dinitrogen are used to shed light on atomic deformations. In the first case, it is usually admitted that the carbon atom must undergo an excitation 
to a state with four unpaired electrons followed by an $\mathrm{sp}^{3}$-like hybridization. In dinitrogen, this kind of promotion is not necessary. The use of EDFs and the comparison of the computed ISBEs with other estimations allow us to follow the evolution of the atomic electronic states in the course of bond formation. As shown, naïve expectations do not necessarily match with our findings, although the deformation of the $\mathrm{C}$ atom turns out to be considerably larger than that of nitrogen. The methane example also serves to show that although small, the $\mathrm{H}-\mathrm{H}$ interaction cannot be neglected, and that its adsorption into the $\mathrm{C}-\mathrm{H}$ bond energy may lie behind several systematic trends in thermodynamic bond energies.

We think that the protocol explained in this contribution provides a theoretically sound definition of intrinsic bond strengths, and that may find its way in the chemical bonding toolbox.

\section{Acknowledgments}

We thank the spanish MINECO/FEDER, grant CTQ2015-65790-P and the FICyT, grant GRUPIN14049.

\section{References}

[1] Braunschweig, H.; Dewhurst, R. D.; Hammond, K.; Mies, J.; Radacki, K.; Vargas, A. Science 2012, 336, 1420.

[2] Köppe, R.; Schnöckel, H. Chem Sci 2015, 6, 1199.

[3] Kalescky, R.; Kraka, E.; Cremer, D. The Journal of Physical Chemistry A 2013, 117, 8981.

[4] Cremer, D.; Wu, A.; Larsson, A.; Kraka, E. Journal of Molecular Modeling 2000, 6, 396.

[5] Shaik, S.; Danovich, D.; Wu, W.; Su, P.; Rzepa, H. S.; Hiberty, P. C. Nature Chemistry 2012, 4, 195.

[6] Frenking, G.; Hermann, M. Angewandte Chemie International Edition 2013, 52, 5922.

[7] Shaik, S.; Rzepa, H. S.; Hoffmann, R. Angewandte Chemie International Edition 2013, 52, 3020. 
[8] Danovich, D.; Shaik, S.; Rzepa, H. S.; Hoffmann, R. Angewandte Chemie International Edition $2013,52,5926$.

[9] Danovich, D.; Hiberty, P. C.; Wu, W.; Rzepa, H. S.; Shaik, S. Chemistry - A European Journal $2014,20,6220$.

[10] Shaik, S.; Danovich, D.; Braida, B.; Hiberty, P. C. Chemistry - A European Journal 2016, 22, 18977.

[11] Frenking, G.; Hermann, M. Chemistry - A European Journal 2016, 22, 18975.

[12] Shaik, S.; Danovich, D.; Braida, B.; Hiberty, P. C. Chemistry - A European Journal 2016, 22, 4116.

[13] Zou, W.; Cremer, D. Chemistry - A European Journal 2016, 22, 4087.

[14] Hermann, M.; Frenking, G. Chemistry - A European Journal 2016, 22, 4100.

[15] Badger, R. M. The Journal of Chemical Physics 1934, 2, 128.

[16] Badger, R. M. The Journal of Chemical Physics 1935, 3, 710.

[17] Kraka, E.; Setiawan, D.; Cremer, D. Journal of Computational Chemistry 2016, 37, 130.

[18] Kaupp, M.; Danovich, D.; Shaik, S. Coordination Chemistry Reviews 2017, 344, 355.

[19] Grunenberg, J. International Journal of Quantum Chemistry 2017, 117, e25359.

[20] Kraka, E.; Larsson, J. A.; Cremer, D. Computational Spectroscopy Wiley-VCH Verlag GmbH \& Co. KGaA: Weinheim, Germany, 2010; p. 105.

[21] Morse, P. M. Physical Review 1929, 34, 57.

[22] Konkoli, Z.; Cremer, D. International Journal of Quantum Chemistry 1998, 67, 1.

[23] Ruedenberg, K. Reviews of Modern Physics 1962, 34, 326.

[24] Bader, R. F. W. Atoms in Molecules: A Quantum Theory; International Ser. of Monogr. on Chem Clarendon Press, 1990. 
[25] Blanco, M. A.; Martín Pendás, A.; Francisco, E. Journal of Chemical Theory and Computation 2005, 1, 1096.

[26] Francisco, E.; Martín Pendás, A.; Blanco, M. A. Journal of Chemical Theory and Computation 2006, 2, 90.

[27] Grabowsky, S.; Genoni, A.; Bürgi, H.-B. Chem Sci 2017, 8, 4159.

[28] Benson, S. W. Journal of Chemical Education 1965, 42, 502.

[29] Martín Pendás, A.; Blanco, M. A.; Costales, A.; Sánchez, P. M.; Luaña, V. Physical Review Letters 1999, 83, 1930.

[30] Lane, J. R.; Contreras-García, J.; Piquemal, J.-P.; Miller, B. J.; Kjaergaard, H. G. Journal of Chemical Theory and Computation 2013, 9, 3263.

[31] Anderson, J. S. M.; Ayers, P. W.; Hernandez, J. I. R. The Journal of Physical Chemistry A 2010, $114,8884$.

[32] Anderson, J. S. M.; Ayers, P. W. The Journal of Physical Chemistry A 2011, 115, 13001.

[33] Srebrenik, S.; Bader, R. F. W. The Journal of Chemical Physics 1974, 61, 2536.

[34] Stone, A. The Theory of Intermolecular Forces; The Theory of Intermolecular Forces Oxford University Press, 2013.

[35] Ponec, R. Journal of Mathematical Chemistry 1997, 21, 323.

[36] Bickelhaupt, F. M.; Baerends, E. J. Reviews in Computational Chemistry, Volume 15, 2000; p. 1.

[37] Parr, R. G.; Yang, W. Density-Functional Theory of Atoms and Molecules; International Series of Monographs on Chemistry Oxford University Press, 1994.

[38] Cancès, E.; Keriven, R.; Lodier, F.; Savin, A. Theoretical Chemistry Accounts: Theory, Computation, and Modeling (Theoretica Chimica Acta) 2004, 111, 373.

[39] Martín Pendás, A.; Francisco, E.; Blanco, M. A. The Journal of Physical Chemistry A 2007, $111,1084$. 
[40] Martín Pendás, A.; Francisco, E.; Blanco, M. A. Phys Chem Chem Phys 2007, 9, 1087.

[41] Giambiagi, M.; de Giambiagi, M. S.; Mundim, K. C. Struc Chem 1990, 1, 423.

[42] Ponec, R.; Bultinck, P.; Gallegos-Saliner, A. J Phys Chem A 2005, 109, 6606.

[43] Mandado, M.; González-Moa, M. J.; Mosquera, R. A. J Comput Chem 2007, 28, 127.

[44] Mundim, K. C.; Giambiagi, M.; de Giambiagi, M. S. J Phys Chem 1994, 98, 6118.

[45] Menéndez, M.; Álvarez Boto, R.; Francisco, E.; Martín Pendás, A. Journal of Computational Chemistry 2015, 36, 833.

[46] Sun, Q.; Berkelbach, T. C.; Blunt, N. S.; Booth, G. H.; Guo, S.; Li, Z.; Liu, J.; McClain, J.; Sayfutyarova, E. R.; Sharma, S.; Wouters, S.; Chan, G. K.-L. submitted to WIREs 2017.

[47] Schmidt, M. W.; Baldridge, K. K.; Boatz, J. A.; Elbert, S. T.; Gordon, M. S.; Jensen, J. H.; Koseki, S.; Matsunaga, N.; Nguyen, K. A.; Su, S.; Windus, T. L.; Dupuis, M.; Montgomery, J. A. Journal of Computational Chemistry 1993, 14, 1347.

[48] Martín Pendás, A.; Francisco, E. Promolden: A QTAIM/IQA code (Available from the authors upon request by writing to ampendas@uniovi.es).

[49] Francisco, E.; Martín Pendás, A. Computer Physics Communications 2014, 185, 2663.

[50] Gallup, G. A. Valence Bond Methods: Theory and Applications; Cambridge University Press, 2002.

[51] Cooper, D. Valence Bond Theory; Theoretical and Computational Chemistry Elsevier Science, 2002.

[52] Shaik, S. S.; Hiberty, P. C. A Chemist's Guide to Valence Bond Theory; Wiley, 2007.

[53] Luaña, V.; Recio, J. M.; Pueyo, L. Phys Rev B 1990, 42, 1791.

[54] Martín Pendás, A.; Blanco, M. A.; Francisco, E. Journal of Computational Chemistry 2009, 30, 98.

[55] Fischer, C. F. Journal of Physics B: Atomic, Molecular and Optical Physics 2006, 39, 2159. 
[56] Voge, H. H. The Journal of Chemical Physics 1936, 4, 581.

[57] Voge, H. H. The Journal of Chemical Physics 1948, 16, 984.

[58] García-Revilla, M.; Francisco, E.; Popelier, P. L. A.; Martín Pendás, A. ChemPhysChem 2013, $14,1211$.

[59] Casals-Sainz, J. L.; Guevara-Vela, J. M.; Francisco, E.; Rocha-Rinza, T.; Martín Pendás, A. ChemPhysChem 2017, 18, 3553.

[60] Martín Pendás, A.; Francisco, E.; Blanco, M. A. The Journal of Chemical Physics 2007, 127, 144103.

[61] Martín Pendás, A.; Francisco, E.; Blanco, M. A. The Journal of Physical Chemistry A 2006, $110,12864$.

[62] Rafat, M.; Popelier, P. L. A. The Quantum Theory of Atoms in Molecules Wiley-VCH Verlag GmbH \& Co. KGaA: Weinheim, Germany, 2007; p. 121.

[63] Francisco, E.; Menéndez Crespo, D.; Costales, A.; Martín Pendás, A. Journal of Computational Chemistry 2017, 38, 816.

[64] Johnston, H. S.; Parr, C. Journal of the American Chemical Society 1963, 85, 2544. 


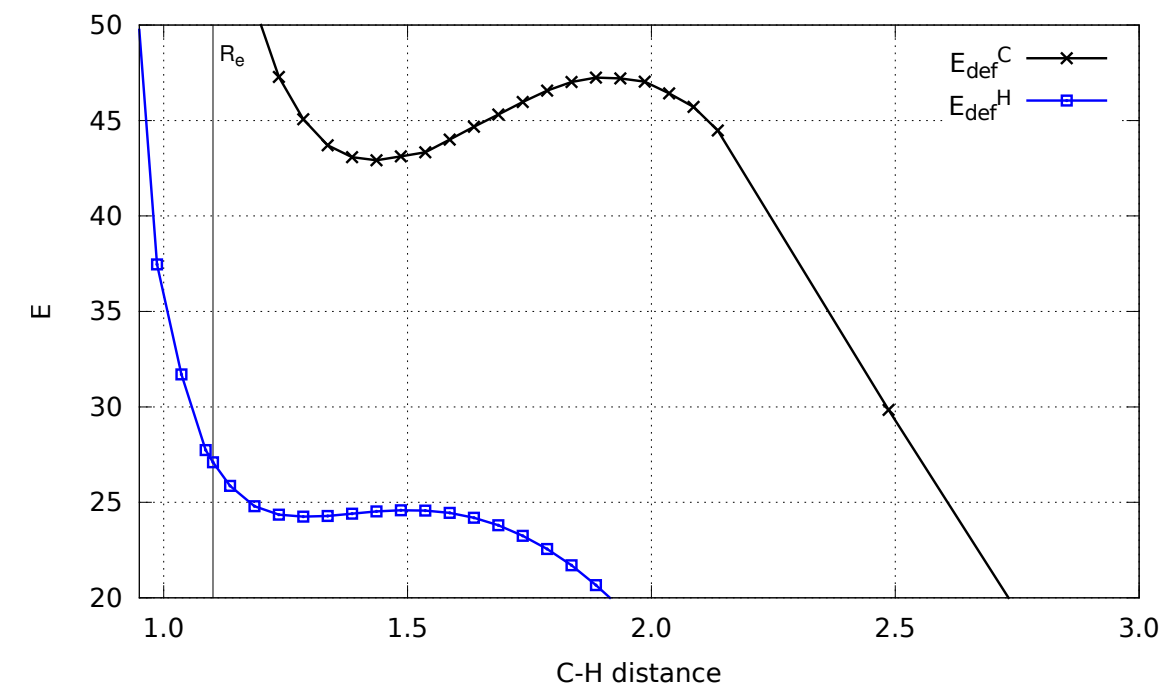

Figure 1: Evolution of the deformation energies of the $\mathrm{C}$ and $\mathrm{H}$ atoms with the $\mathrm{C}-\mathrm{H}$ distance. The vertical lines marks the computed equilibrium geometry. Distances in $\AA$ and energies in $\mathrm{kcal} / \mathrm{mol}$

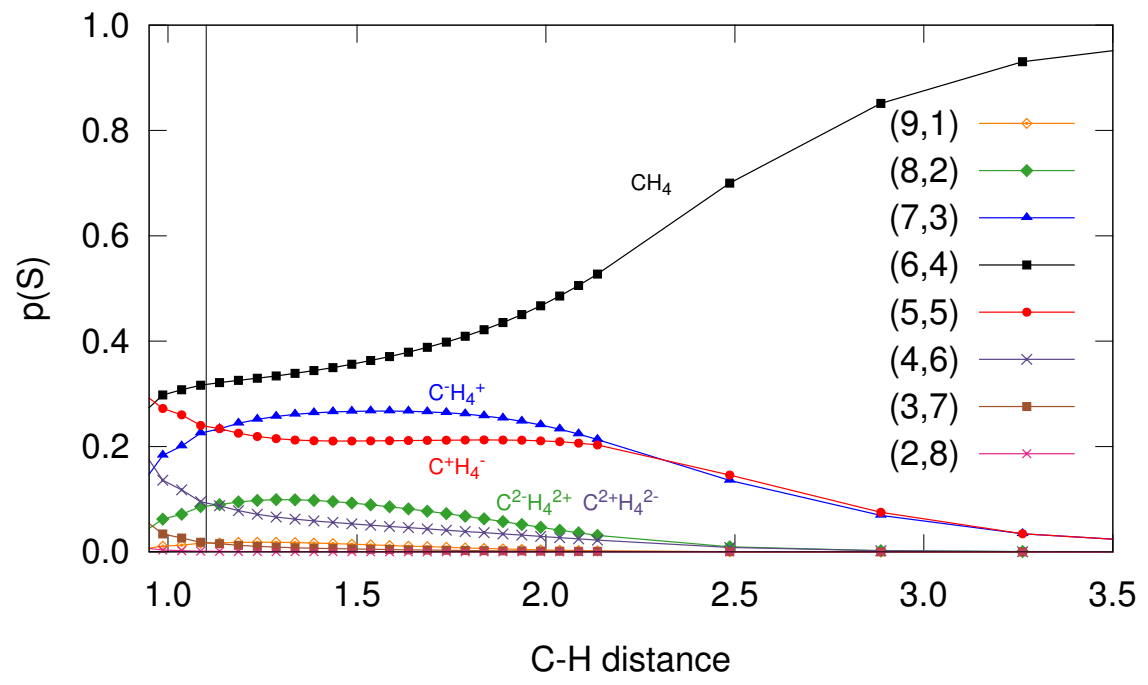

Figure 2: Evolution of the two-fragment spinless EDF. $p(S)$ is the probability of each resonance structure $S=\left(n_{C}, n_{4 H}\right)$. The vertical lines marks the computed equilibrium geometry. Distances in $\AA$ 

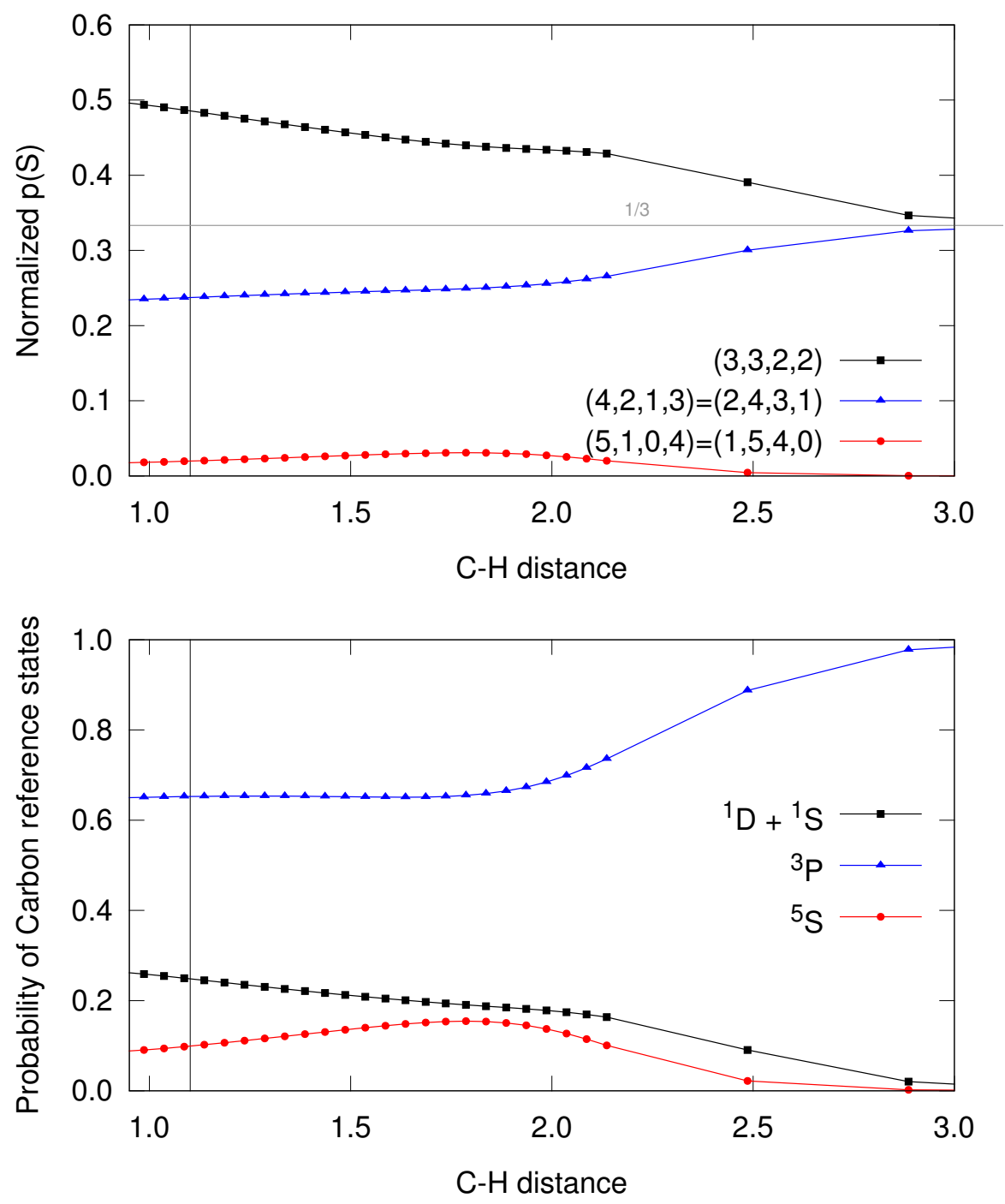

Figure 3: Top (a): evolution of the normalized spin-resolved EDF corresponding to a neutral $C$ atom. The notation is $\mathrm{S}=\left(\mathrm{n}_{\mathrm{C}}^{\alpha}, \mathrm{n}_{\mathrm{C}}^{\beta}, \mathrm{n}_{\mathrm{H}}^{\alpha}, \mathrm{n}_{\mathrm{H}}^{\beta}\right)$. Down (b): Probabilities of the different spin multiplets of the neutral $\mathrm{C}$ atom in-the-molecule. See the text for details. All distances in $\AA$ 


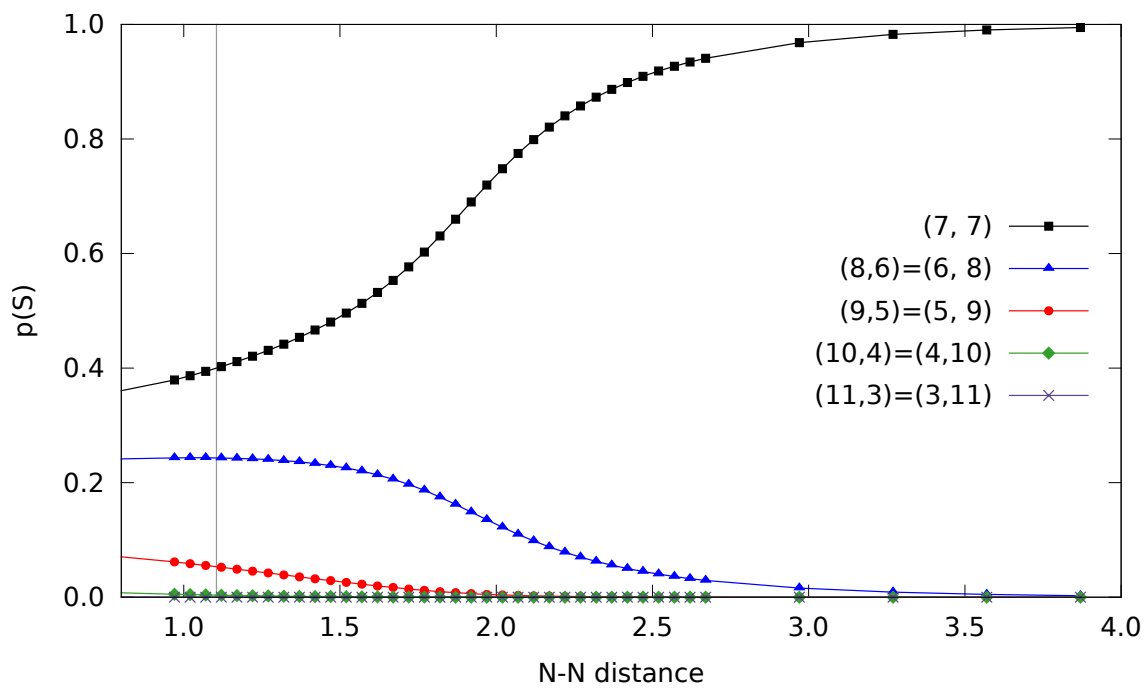

Figure 4: Evolution of the two-fragment spinless EDF in dinitrogen. $p(S)$ is the probability of each resonance structure $S=\left(n_{N 1}, n_{N 2}\right)$. The vertical lines marks the computed equilibrium geometry. Distances in $\AA$ 

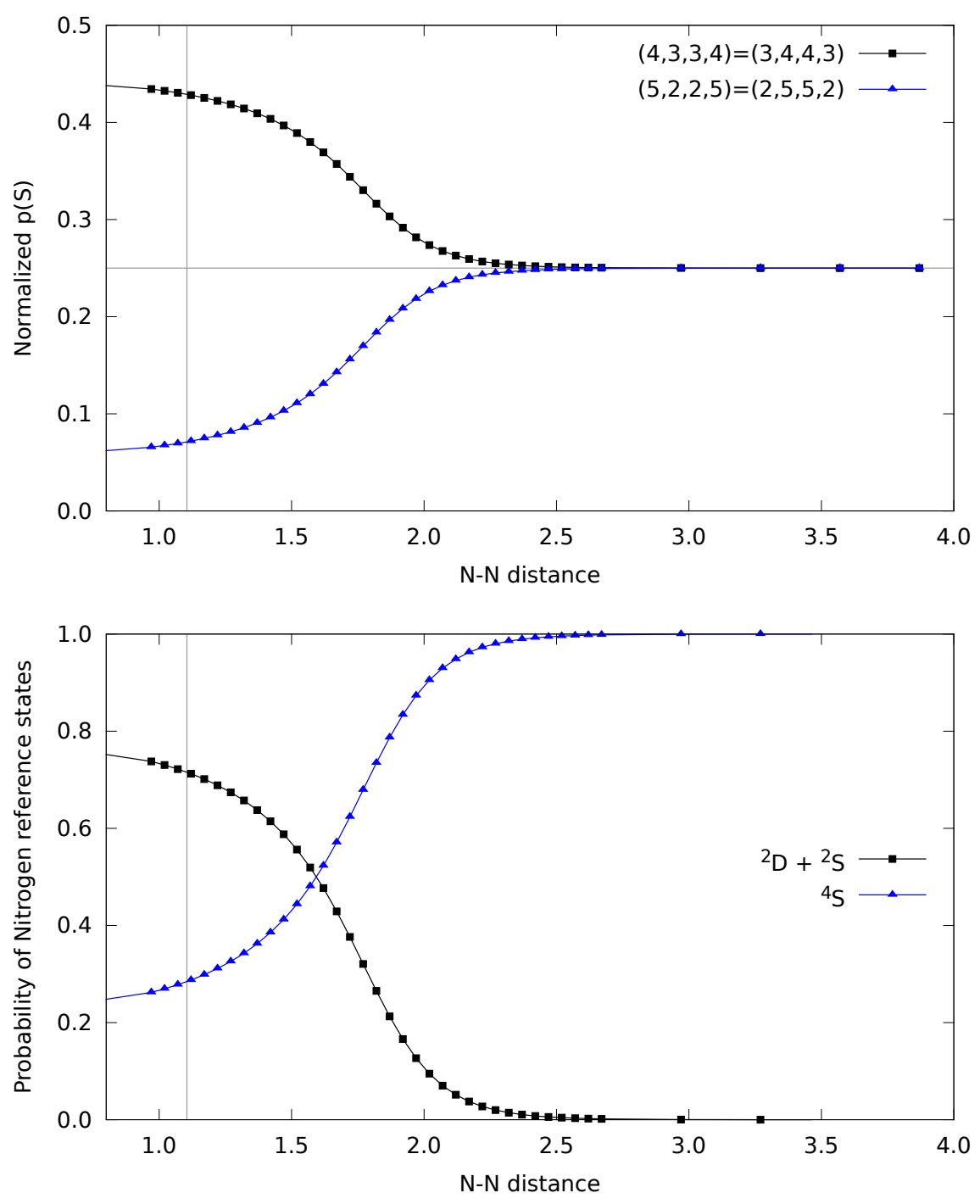

Figure 5: Top (a): evolution of the normalized spin-resolved EDF corresponding to a neutral $\mathrm{N}$ atom in dinitrogen. The notation is $S=\left(n_{\mathrm{N} 1}^{\alpha}, n_{\mathrm{N} 1}^{\beta}, \mathrm{n}_{\mathrm{N} 2}^{\alpha}, \mathrm{n}_{\mathrm{N} 2}^{\beta}\right)$. Down (b): Probabilities of the different spin multiplets of the neutral $\mathrm{N}$ atom in-the-molecule. See the text for details. All distances in $\AA$ 


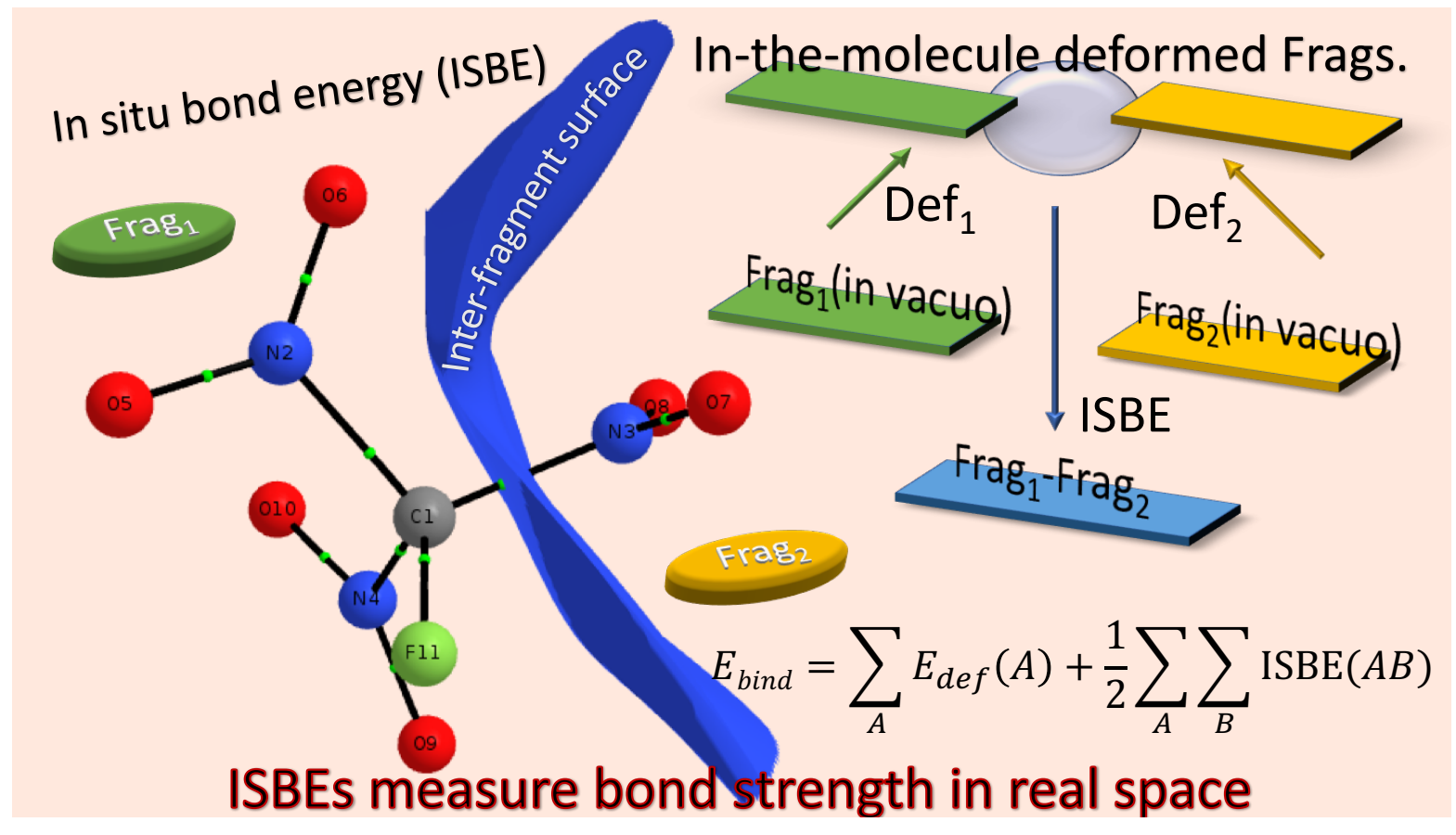

Figure 6: *

Table of Contents Graphic: A rigorous definition of intrinsic bond strength based on the partitioning of a molecule into real space fragments, amenable to experimental determination, is presented. 